\title{
Mechanische Revaskularisation beim Schlaganfall
}

\section{Qual oder Wahl}

\section{Die mechanische Rekanalisation eines akuten Verschlusses eines Hirn- gefäßes avancierte in den letzten Jahren bei einem bestimmten, spe- ziell selektionierten Patientenklien- tel zur Therapie der Wahl. Stellenwei- se exzellente Behandlungsergebnis- se untermauerten die Indikation zur mechanischen Wiedereröffnung ver- schlossener Hirngefäße.}

\section{„Qual der Wahl“}

Die Qual liegt derzeit darin, dass eine Vielzahl mechanischer Systeme zur Auswahl stehen und der Druck vonseiten der klinischen Kollegen groß ist, gute Ergebnisse zu erzielen, da bis heute randomisierte Studien, die die Effizienz der Behandlung beweisen, fehlen. In dieser Arbeit werden die einzelnen Systeme unter Berücksichtigung ihrer Effizienz dargestellt. Einzelne neuere Studien zeigen überwiegend gute Ergebnisse bei der Behandlung schwerer Schlaganfälle, bei denen insbesondere die etablierte und weit verbreitete systemische Lysebehandlung versagt $[11,16]$. Ausschlaggebend sind die richtige Auswahl der Patienten und folgerichtig die Zuordnung in die richtige Behandlungsgruppe, also konservativ, invasiv mittels IVT (intravenöse Lystherapie) oder LIF (lokale intraarterielle Fibrinolyse). Es ist ein Trugschluss zu glauben, dass mit der Rekanalisation, die statistisch gesehenen für den klinischen Erfolg entscheidend ist [12, 15, 20], der Therapieerfolg automatisch gegeben ist. Die neuen mechanischen Syste- me erlauben eine Rekanalisation in bis zu 90\% der Fälle und zeigen trotzdem nicht immer die erhofften klinischen Resultate. Es bleiben die Qual und Wahl der richtigen Indikation als auch der richtigen Behandlungsform.

\section{Endovaskuläre Schlaganfall- behandlung - Rückblick}

In Aachen wurde 1979 die erste intraarterielle Lysebehandlung bei einem kurzstreckigen Basilarisverschluss erfolgreich durchgeführt [21]. Seit mehr als 10 Jahren ist die lokale intraarterielle Fibrinolyse (LIF) ein anerkanntes und etabliertes Verfahren zur Behandlung proximaler Hirngefäßverschlüsse. Wegweisend hierfür war Ende der 90er Jahre die PROACT-II-Studie, bei der die intraarterielle Thrombolyse mit Prourokinase innerhalb von $6 \mathrm{~h}$ sich als sicher und effektiv herausstellte und zu einer signifikant höheren Rate funktionell unabhängiger Patienten führte, insbesondere bei initial schwerer Schlaganfallsymptomatik und proximalem Gefäßverschluss [6]. In der PROACT-II-Studie war der klinische Outcome nach 90 Tagen mit mRs 0-2 („modified Rankin scale" $0-2$, kein oder geringes neurologisches Defizit) bei Mediahauptstammverschluss in der mit Prourokinase behandelten Gruppe mit $40 \%$ bei einer Rekanalisationsrate von $67 \%$ deutlich besser als mit 25\% in der der mit Heparin allein behandelten Gruppe.

Es folgten so genannte Bridgingbehandlungen, wobei nach Ausschluss einer
Blutung in der zerebralen Schnittbildgebung innerhalb von zunächst $3 \mathrm{~h}$ und seit der ECASS-III-Studie innerhalb von 4,5 h unmittelbar mit einer systemischen Behandlung begonnen wurde, um dann bei fehlender klinischer Besserung die Therapie endovaskulär fortzusetzen. Neben der Kombination aus intravenös und intraarteriell appliziertem rt-PA (rekombinanter Gewebeplasminogenaktivator) wird auch eine Kombination aus intravenös appliziertem GPIIa/IIIb-Hemmer und intraarteriellem rt-PA eingesetzt $[4,5]$.

Die endovaskulären Behandlungen beim akuten Schlaganfall sind bis 2008 nur in wenigen Kliniken in Deutschland mit hohen Fallzahlen verbunden gewesen. In - Abb. 1 werden die Fallzahlen am Universitätsklinikum Freiburg von 1997 bis 2007 und am Klinikum Augsburg von 2008 bis 2012 dargestellt. Dies zeigt, dass an 2 unterschiedlichen Standorten sich jeweils die endovaskuläre Therapie erst durchsetzen musste. Die Etablierung ist eng verbunden mit der lokalen und überregionalen Infrastruktur, erfolgreichen Behandlungen sowie einem engen, vertrauensvollen und interdisziplinären Zusammenarbeiten von Neuroradiologen oder Radiologen und Neurologen. Ausschlaggebend für die niedrigen Behandlungszahlen war und ist der Tatsache geschuldet, dass rt-PA aufgrund der Ergebnisse der NINDS-Studie 1995 bis heute als Standardtherapie gilt. 


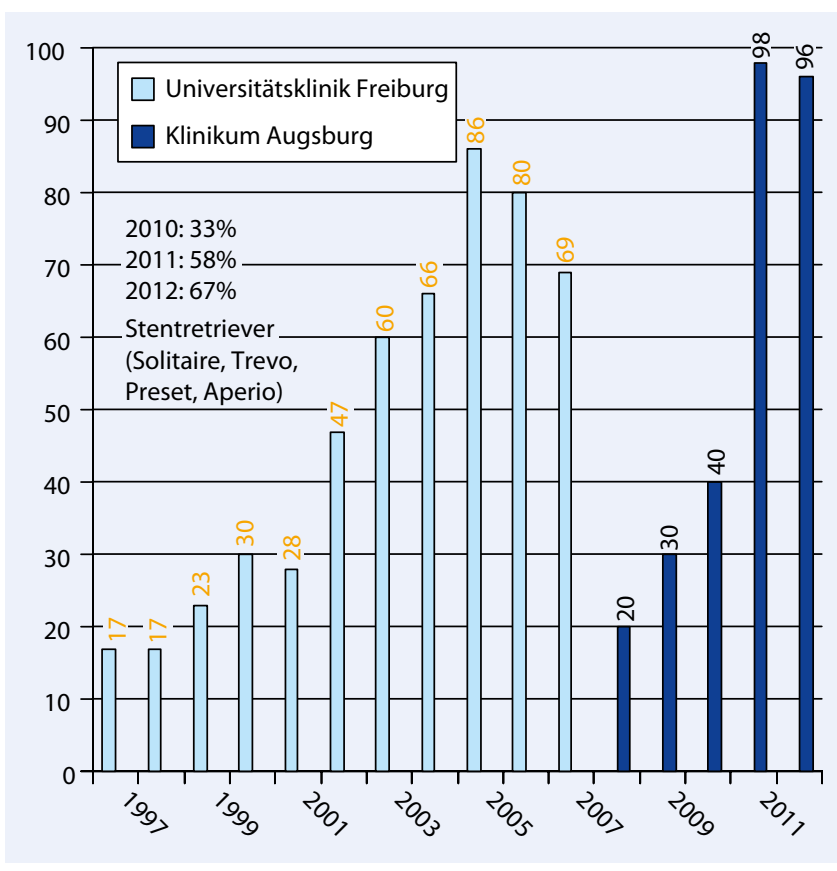

schiedenen Fachdisziplinen wie Anästhesie, Neuroradiologie und Neurologie mit ein und muss, um eine zeitnahe Behandlung im Sinne von „time is brain“ zu gewährleisten, von einer Klinik mit einem neurovaskulären Anspruch gestellt werden. In großen Stroke Units werden heutzutage in Europa Thrombolyseraten zwischen 15 und 25\% erreicht. In Augsburg wurden von in den letzten beiden Jahren 1500-1700 stationär behandelten Schlaganfallpatienten etwa $18 \%$ der Patienten behandelt, davon $1 / 3$ endovaskulär und $2 / 3$ systemisch. Nicht vergessen sollte man, dass man für die verbleibenden $80 \%$ ebenfalls eine adäquate Diagnostik anbieten muss, die ebenfalls radiologisches Personal und Gerätschaften wie CT oder MR bindet.

\section{Mechanische Rekanalisation - Rückblick}

\section{Etablierung der endovaskulären Behandlung}

Die Limitationen von rt-PA werden durch das relativ enge zeitliche Fenster von $4,5 \mathrm{~h}$, durch die Verschlusslokalisation und nicht zuletzt durch die Thrombuslast offenbar. Die Akzeptanz der endovaskulären Therapie wurde durch die von Mattle et al. [11] in Stroke 2008 publizierte Vergleichsarbeit von IVT und LIF bei Mediahauptstammverschlüssen untermauert. In dieser Arbeit konnte ein eindeutiger Vorteil der LIF mit Urokinase gegenüber der IVT bei einem vergleichbaren Patientenklientel mit Mediahauptstammverschluss, der bildgebend durch ein „dichtes Mediazeichen" nachgewiesen wurde, herausgearbeitet werden. Ein guter klinischer Verlauf mit $m R s \leq 2$ zeigt sich bei nahezu $30 \%$ mehr Patienten mit LIF als bei IVT. Hinzu kommt, dass bei einer Thrombuslänge von 7-8 mm im Mediahauptstamm keine Rekanalisation durch IVT zu erwarten ist [16], sodass bei entsprechender Thrombuslast folgerichtig die intraarterielle Behandlung angestrebt werden sollte.

Die intraarterielle Rekanalisation wird in den kommenden Jahren nicht zuletzt auch aufgrund der demographischen Entwicklung immer häufiger angewendet werden. Nach einer Umfrage der Deutschen Gesellschaft für Neuroradiologie werden aktuell ca. 85\% der Fläche der
Bundesrepublik durch Zentren mit einem Einzugsbereich von $60 \mathrm{~km}$ abgedeckt. Die Therapie ist prozedural nicht allzu kompliziert und kann relativ rasch erlernt werden. Um die Qualität zu sichern, ist aber unbedingt erforderlich, dass dies ein Routineeingriff ist, der eine bestimmte Fallzahl pro Jahr voraussetzt. Die Gefahren sind vielfältig und reichen von falscher Indikationsstellung zur Behandlung und damit verbundenem schlechtem Outcome bis zum fehlenden Beherrschen des Komplikationsmanagements bei Eingriffen an den Hirngefäßen. Konsequenterweise sollten Patienten mit schwerem Schlaganfall aus einem Haus ohne Expertise entsprechend der 2010 in Stroke publizierten Arbeit von Pfefferkorn et al. [14] in ein neurovaskuläres Zentrum verbracht werden.

\section{Qual oder Wahl - Schlaganfälle kommen immer zu Unzeiten}

Es ist nicht nur gefühlt so, dass Schlaganfallpatienten sich selten an die Regelarbeitszeiten halten. In Augsburg wurden im Jahr 2012 akute ischämische Schlaganfälle in $58 \%$ der Fälle außerhalb der regulären Dienstzeiten endovaskulär behandelt. Dies erfordert ein schlagkräftiges Team über $24 \mathrm{~h}$ und an 7 Tagen in der Woche. Diese Infrastruktur schließt Ärzte, MTRA und Pflegekräfte aus den ver-
Seit Ende der 90er Jahre werden mechanische Systeme zur Verbesserung der Rekanalisationsergebnisse eingesetzt. Die Limitationen der lokalen Lyse liegen in der Zeit bis zum Erreichen der Rekanalisation mit durchschnittlich $1,5 \mathrm{~h}$ und in der Rate erfolgreicher Rekanalisationen mit durchschnittlich $63 \%[4,5,12,15]$. Es wurden verschiedene Systeme mit unterschiedlichen Ansätzen getestet. Aufgrund der unterschiedlichen Wirkmechanismen kann man diese in proximale und distale Systeme einteilen. Bei den distalen Systemen wird zunächst der Thrombus passiert und dieser dann von distal nach proximal behandelt. Hauptvertreter hierbei sind die Stentretriever, die distal des Thrombus beginnend geöffnet werden und den Thrombus nach proximal überbrücken. Dieser Bypass ermöglicht eine zumindest vorübergehende Reperfusion, bis je nach Konsistenz des Thrombus dieser die Stentstreben durchwandert und das Gefäß erneut verschließt. Bei den proximalen Systemen wird das Blutgerinnsel von proximal kommend entfernt. Vertreter dieser Systeme sind Absaugen mit dem Penumbrasystem, der Einsatz einer Greifzange (Alligator Device, Chestnut Medical Technologies, Menlo Park, CA, USA) oder dem endovasalen Ultraschallverfahren (EKOS, MikroLysUS Infusen Cathe- 
ter, EKOS Corporation, Bothwell, WA, USA).

Eine andere Einteilung bezieht sich auf die Systeme, wobei man wie in $\bullet$ Tab. 1 Draht-, Aspirations-, Zertrümmerungssysteme und PTA sowie Stentsysteme unterscheiden kann. Die Wirkungsweise bei Thrombuszertrümmerung durch Mikrodrahtmanipulation oder durch das EKOS-System liegt in einer Oberflächenvergrößerung des Blutgerinnsels, damit sowohl intrinsische als auch exogen zugeführte Fibrinolytika besser wirken.

Die unterschiedlichen Behandlungsoptionen münden in ein multimodales Behandlungskonzept, das unterschiedliche Applikationen von Fibrinolytika und unterschiedliche mechanische Rekanalisationsverfahren von Saugen, Stentrieven bis PTA und Stenting $[1,2,3,4,5,7,8,9$, $10,13,15,17,18,19,20]$ umfasst. Vorteil der mechanischen Verfahren ist, dass diese am effektivsten in Bezug auf Rekanalisation [15] sind und zudem auch bei Patienten mit Kontraindikationen für Fibrinolytika eingesetzt werden können. Gemeinsam ist diesen Systemen, dass sie eine hohe Effizienz zur Rekanalisation aufweisen, die von $55-90 \%$ reicht.

\section{Mechanische Rekanalisation - Update und Qual der Wahl}

Die mechanische Rekanalisation hat in ihren Anfängen nicht die gewünschten klinischen Ergebnisse geliefert. Trotzdem haben 2004 das Merci- und 2008 das Penumbrasystem die FDA-Zulassung erhalten. Lagen in der initialen Penumbrastudie die Rekanalisationsraten noch bei $100 \%$, konnte in der folgenden Studie bei Verwendung dieses "Absaugkatheters“ bei 125 Patienten immer noch eine Rekanalisationsrate von $82 \%$ erreicht werden [2]. Diese exzellenten Daten wurden vom klinischen Verlauf mit nur 25\% mit einem mRS von $\leq 2$ und einer Letalität von 33\% nach 90 Tagen erheblich getrübt. In der aktuell laufenden randomisierten THERAPIE-Studie wird das Penumbrasystem nochmals überprüft und die Indikation zur Behandlung kritischer gestellt.

Im Jahr 2006 wurden die ersten Behandlungen mit Stents oder Stents und PTA beim akuten Schlaganfall veröffentlicht. In einer Multicenteranalyse

\section{A. Berlis \\ Mechanische Revaskularisation beim Schlaganfall. Qual oder Wahl}

Zusammenfassung

Klinisches/methodisches Problem. Der akute ischämische Schlaganfall ist die dritthäufigste Todesursache und der häufigste Grund für eine Behinderung. Aufgrund der demographischen Entwicklung ist zukünftig mit einem Fallzahlanstieg zu rechnen. Radiologische Standardverfahren. Die Auswahl der zu behandelnden Patienten erfolgt mittels unterschiedlicher diagnostischer CTund MR-Modalitäten. Die Behandlung erfolgt in der Regel in einer biplanen Angiographieanlage

Methodische Innovationen. Die intraarterielle lokale Fibrinolyse (LIF) hat sich als im Hinblick auf Rekanalisation und klinischem Verlauf als effektiv herausgestellt. Mechanische Systeme werden seit Ende der 90er Jahre eingesetzt. Die erst seit wenigen Jahren zum Einsatz kommenden Stentretriever haben sich als hoch effektiv herausgestellt.
Leistungsfähigkeit. Mit mechanischen Verfahren können in bis zu 90\% der Hirngefäße wiedereröffnet werden.

Bewertung. Die Stentretriever haben bislang gegenüber den bisherigen mechanischen Verfahren zu besseren klinischen Verläufen geführt.

Empfehlung für die Praxis. Stentretrieving ist die Methode der ersten Wahl bei der Wahl mechanischer Verfahren zur intraarteriellen Rekanalisationsbehandlung beim akuten Schlaganfall.

\section{Schlüsselwörter Biplane Angiographieanlage . Endovaskuläre Behandlung . Mechanische Systeme . Systemische Lysebehandlung . Stentretrieving}

\section{Mechanical recanalization in acute stroke. Torture or choice}

\begin{abstract}
Clinical/methodical issue. Stroke is the third most common cause of death in the developed world and the leading cause of disability worldwide. The present demographic development will lead to increased numbers of stroke patients in the future.

Standard radiological methods. Patient selection takes place by using various computer tomographic (CT) or magnetic resonance (MR) imaging modalities. For endovascular stroke treatment a modern biplane angiography suite is recommended. Methodical innovations. Intra-arterial chemical thrombolysis was shown to be effective in achieving recanalization and improving clinical outcome. Mechanical devices were introduced in the late 1990s and the highly effective stent retriever was recently included.
\end{abstract}

mit 1122 Patienten konnten die Effektivität und hohe Rekanalisationsraten nachgewiesen werden [8]. Die Nachteile liegen in der Notwendigkeit einer doppelten Thrombozytenaggregationshemmung, die bei wieder entfernbaren selbstexpandierbaren Stents, so genannten Stentretrievern, nicht notwendig ist.

Aktuell werden nahezu 10 verschiedene Stentretriever von verschiedenen Fir-
Performance. Mechanical devices allows a recanalization rate of up to $90 \%$ of affected brain vessels.

Achievements. The advantage of a stent retriever compared to other mechanical devices seems to be an improvement in good clinical outcome.

Practical recommendations. Currently, the stent retriever seems to be the definitive first choice in the use of mechanical devices for intra-arterial recanalization treatment in acute ischemic stroke.

\section{Keywords}

Biplane angiography suite - Endovascular treatment · Mechanical systems · Systemic lysis treatment $\cdot$ Stent retriever men angeboten, wobei aktuell Systeme wie Solitaire der Firma ev3-covidien und Trevo der Firma Stryker international am häufigsten eingesetzt werden. Dies spiegelt sich auch in der Zulassung durch die FDA und CE-Markierung sowie den in PubMed gelisteten 49 Publikationen für Solitaire und 24 für Trevo wider. Bestimmend für den aktuell dominierenden Einsatz von Stentretrievern waren die zuletzt 


\begin{tabular}{|c|c|c|c|c|}
\hline & System & Funktion & Einteilung & Indikation/Anmerkungen \\
\hline \multirow[t]{4}{*}{$\begin{array}{l}\text { Draht- } \\
\text { systeme }\end{array}$} & Gewöhnlicher Mikrodraht & $\begin{array}{l}\text { Thrombusfraktionierung, Oberflächen- } \\
\text { vergrößerung für medikamentöse } \\
\text { Behandlung }\end{array}$ & Proximal & $\begin{array}{l}\text { Sondieren intrakranieller Gefäße } \\
\text { durch einen Mikrokatheter }\end{array}$ \\
\hline & $\begin{array}{l}\text { Drahtschlinge „Snare“ } \\
\text { (Firma Covidien/ev3, Dublin, Ireland) }\end{array}$ & $\begin{array}{l}\text { Bergen oder Fraktionierung von } \\
\text { Blutgerinnseln }\end{array}$ & Proximal & $\begin{array}{l}\text { Bergen von Fremdkörpern, } \\
\text { z. B. Platinspiralen }\end{array}$ \\
\hline & $\begin{array}{l}\text { Körbchen, z. B. Catch } \\
\text { (Fa. Balt, Frankreich) }\end{array}$ & Bergen von Blutgerinnseln & Distal & Bergen von Fremdkörpern \\
\hline & $\begin{array}{l}\text { MERCI-System (Fa. Stryker Neuro- } \\
\text { vascular, Mountain View, CA, USA) }\end{array}$ & $\begin{array}{l}\text { Bergen von Blutgerinnseln } \\
\text { Korkenzieherähnliche Drahtkonfiguration }\end{array}$ & Distal & Bergen von Blutgerinnseln \\
\hline \multirow[t]{4}{*}{$\begin{array}{l}\text { Thrombus- } \\
\text { aspiration }\end{array}$} & $\begin{array}{l}\text { Proboscis OTW-Mikrokatheter } \\
\text { (Fa. Medical Braiding) }\end{array}$ & Absaugen & Proximal & Mikrokatheter \\
\hline & Penumbra (Fa. Penumbra) & $\begin{array}{l}\text { Absaugen mittels Pumpe und } \\
\text { Thrombusentfernung }\end{array}$ & Proximal & $\begin{array}{l}\text { Sicherheitsstudie 10/2005- 02/2006 } \\
\text { (20 Patienten), Folgestudie mit } 125 \\
\text { Patienten ab 05/2006 }\end{array}$ \\
\hline & X-Sizer (Fa. ev3) & Rotationssystem zum Absaugen & Proximal & Studie nach 2 Patienten eingestellt \\
\hline & Angiojet (Fa. Possis) & $\begin{array}{l}\text { Absaugen durch gepulste Hochdruck- } \\
\text { wasserpumpe }\end{array}$ & Proximal & Machbarkeitsstudie \\
\hline \multirow[t]{3}{*}{$\begin{array}{l}\text { Thrombus- } \\
\text { zertrümme- } \\
\text { rung }\end{array}$} & $\begin{array}{l}\text { EKOS (Fa. EKOS Corporation, } \\
\text { Bothwell, WA, USA) }\end{array}$ & $\begin{array}{l}\text { Endovaskuläre Ultraschallanwendung } \\
\text { mit Thrombuszertrümmerung und rt-PA- } \\
\text { Gabe }\end{array}$ & Proximal & $\begin{array}{l}\text { Machbarkeitsstudie, Anwendung im } \\
\text { Rahmen IMS-II-und-III-Studie (EKOS } \\
\text { + Bridging) }\end{array}$ \\
\hline & LATIS (Fa. Latis, CA, USA) & Direkter Laserbeschuss des Blutgerinnsels & Proximal & Sicherheitsstudie, eingestellt \\
\hline & EPAR (Fa. Endovasix, CA, USA) & $\begin{array}{l}\text { Laserinduzierte Zertrümmerung } \\
\text { innerhalb der Katheterspitze }\end{array}$ & Distal & $\begin{array}{l}\text { Sicherheits- und Machbarkeitsstudie, } \\
\text { eingestellt }\end{array}$ \\
\hline $\begin{array}{l}\text { Stent- } \\
\text { retriever }\end{array}$ & $\begin{array}{l}\text { Unter anderen Solitaire (Firma } \\
\text { Covidien/ev3), Trevo (Firma Stryker), } \\
\text { Aperio (Firma Acandis, Deutschland) } \\
\text { oder Preset (Firma Phenox, Deutsch- } \\
\text { land) }\end{array}$ & $\begin{array}{l}\text { Zurückziehbare selbstexpandierbare } \\
\text { Stents }\end{array}$ & Distal & $\begin{array}{l}\text { Aktuell die wahrscheinlich am } \\
\text { häufigsten eingesetzten Systeme zur } \\
\text { mechanischen Rekanalisations- } \\
\text { behandlung }\end{array}$ \\
\hline $\begin{array}{l}\text { PTA und } \\
\text { Stent }\end{array}$ & Ballons und Stents & $\begin{array}{l}\text { Rekanalisation bei arteriosklerotischer } \\
\text { Stenose bzw. Verschluss mit Thrombus- } \\
\text { verlagerung in die Gefäßwand }\end{array}$ & Distal & $\begin{array}{l}\text { Ballonexpandierbarer Stent (Pharos, } \\
\text { Fa. Micrus), selbstexpandierbarer Stent } \\
\text { (Wingspan, Firma Stryker) für Hirnge- } \\
\text { fäßstenosen zugelassen, PTA-Ballons } \\
\text { für Stenosen (Zulassung z. B. für Gate- } \\
\text { wayballon für Hirngefäßstenosen) }\end{array}$ \\
\hline
\end{tabular}

publizierten Vergleichsarbeiten, die die Stentretriever Trevo und Solitaire mit dem Merci-System verglichen haben [13, 19]. Beim Vergleich Merci mit Trevo waren die Trevo-Ergebnisse deutlich besser mit $68 \%$ mit TICI-2b- und -3-Rekanalisation und $40 \%$ gutem Outcome nach 90 Tagen mit mRs 0-2 gegenüber dem Merci-System mit 44 und 21,8\%. Diese Ergebnisse wurden in der SWIFT-Vergleichsstudie zwischen Solitaire und Merci mit gutem klinischem Outcome nach 90 Tagen mit 58 vs. $33 \%$ bestätigt. In $\bullet$ Abb. 2 wird ein klinisches Beispiel vorgestellt.

Die Ergebnisse für das Merci- als auch das Penumbrasystem sind ernüchternd und sprechen nicht für eine erfolgversprechende zukunftweisende Methode. Dies wird auch durch die im April 2012 publik gewordene Mitteilung, dass in den USA die IMS-III-Studie abgebrochen wurde, bestärkt [9]. In dieser Studie erfolgte eine Bridgingbehandlung mit Verwendung mechanischer Systeme, wobei der endovaskuläre Arm schlechter als die IVT abschnitt. Die Ursache liegt möglicherweise darin begründet, dass als mechanische Systeme EKOS, Penumbra, v. a. Merci und nur wenige Solitaire verwendet wurden.

Es bleibt abzuwarten, wie weitere und v. a. randomisierte Studien mit Stentretrievern die zukünftigen Behandlungsstrategien bestimmen werden. Die jüngsten Ergebnisse und die zunehmenden Erfahrungen der Anwender lassen positives erwarten.

\section{Fazit für die Praxis}

- Die mechanischen Systeme zur endovaskulären Rekanalisationsbehandlung sind hocheffektiv bzgl. der Rekanalisationsrate und scheinen auch mit deutlich besseren klinischen Verläufen einherzugehen.

- Mit Stentretrievern können aktuell und im Vergleich mit bisherigen Systemen die besten Ergebnisse erzielt werden.

- Nicht zu vergessen bleibt, dass das Wichtigste die richtige Therapieentscheidung ist, die durch die bildgebende Diagnostik maßgeblich beeinflusst wird. 

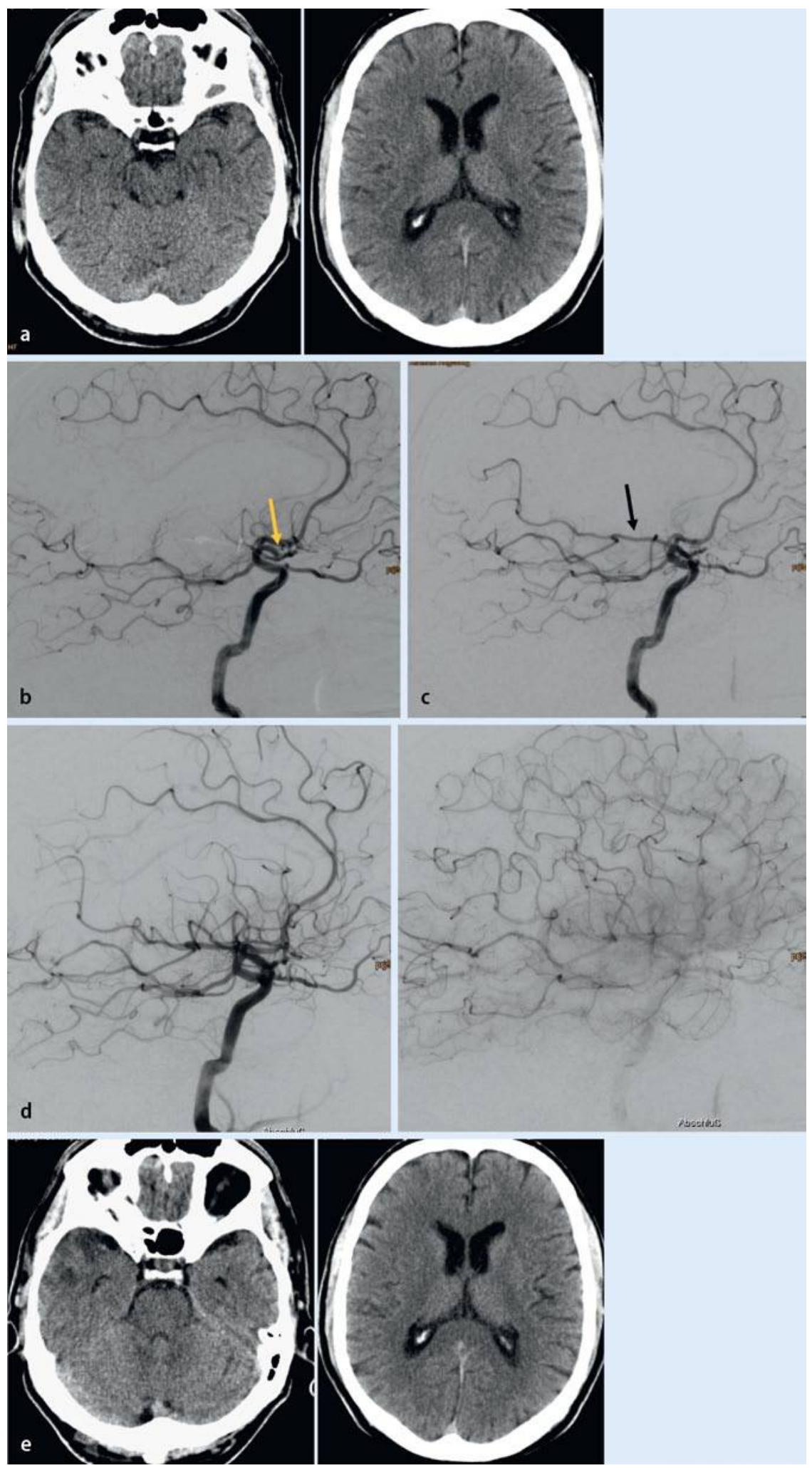

Abb. $2 \Delta 66$ Jahre alter Patient mit Hemiplegie links bei distalem Mediahauptstammverschluss rechts (a). Unmittelbar nach dem CT erfolgt der Beginn der Therapie mit $50 \mathrm{mg}$ rt-PA i.v., gefolgt von $20 \mathrm{mg}$ rt-PA ia bei relativ kurzem Verschluss (b). Bei fehlender Rekanalisation Entscheidung für den Einsatz eines Stentretrievers (Solitaire FR 4×20, Fa. Covidien/ev3, USA, c). Nach einmaligem Retrieving Rekanalisationsergebnis entsprechend $\mathrm{TICl} 2 \mathrm{~b}$ (d). Im postoperativen $\mathrm{CT}$ lediglich kleiner umschriebener Infarkt temporopolar rechts

\section{Korrespondenzadresse}

Prof. Dr. A. Berlis

Klinik für Diagnostische

Radiologie und Neuro-

radiologie, Klinikum Augsburg,

Stenglinstr. 2, 86156 Augsburg

Ansgar.berlis@

klinikum-augsburg.de

Interessenkontlikt. Der korrespondierende Autor weist auf folgende Beziehungen hin: Proctor- und Beratervertrag mit der Firma ev3/Covidien.

\section{Literatur}

1. Berlis A, Lutsep H, Barnwell S et al (2004) Mechanical thrombolysis in acute ischemic stroke with EPAR (Endovascular Photoacoustic Recanalization). Stroke 35:1112-1116

2. Bose A, Alfke K, Henkes H et al (2008) The penumbra system: a mechanical device for the treatment of acute stroke due to thromboembolim. AJNR Am J Neuroradiol 29:1409-1413

3. Dorn F, Stehle S, Lockau H et al (2012) Endovascular treatment of intracerebral artery occlusions with the solitaire stent: single-centre experience with 108 recanalization procedures. Cerebrovasc Dis 34:70-77

4. Eckert B (2009) Acute stroke therapy 1981-2009. Clin Neuroradiol1:8-19

5. Eckert B, Koch B, Thomalla G et al (2005) Aggressive therapy with intravenous abciximab and intraarterial rt-PA and additional PTA/stenting improves clinical outcome in acute vertebrobasilar occlusion: combined local fibrinolysis and intravenous abciximab in acute vertebrobasilar stroke treatment (FAST): results of a multicenter study. Stroke 36:1160-1165

6. Furlan A, Higashida R, Wechsler L et al (1999) Intraarterial prourokinase for acute ischemic stroke: the PROACT II study: a randomised controlled trial. JAMA 282:2003-2011

7. Galimanis A, Jung S, Mono M-L et al (2012) Endovascular therapy of 623 patients with anterior circulation stroke. Stroke 43:1052-1057

8. Gupta R, Tayal AH, Levy El et al (2011) Intra-arterial thrombolysis or stent placement during endovascular treatment for acute ischemic stroke leads to the highest recanalization rate: results of a multicenter retrospective study. Neurosurgery 68:16181623

9. Interventional Management of Stroke (IMS) III Trial (IMSIII). This study is ongoing, but not recruiting participants. ClinicalTrials.gov Identifier: NCT00359424; First received: July 31, 2006; Last updated: April 27, 2012

10. Koh JS, Lee SJ, Ryu CW, Kim SH (2012) Safety and efficacy of mechanical thrombectomy with solitaire stent retrieval for acut ischemic stroke: a systematic review. Neurointervention 7:1-9

11. Mattle HP, Arnold M, Georgiadis D et al (2008) Comparison of intra-arterial and intravenous thrombolysis for ischemic stroke with hyperdense middle cerebral artery sign (HMCAS). Stroke 39:379-383 
12. Mazighi M, Serfaty JM, Labreuche J (2009) Comparison of intravenous alteplase with a combined intravenous-endovascular approach in patients with stroke and confirmed arterial occlusion (RECANALISE study): a prospective cohort study. Lancet Neurol 8:802-809

13. Nogueira RG, Lutsep HL, Gupta R et al (2012) Trevo versus merci retrievers for thrombectomy revascularisation of large vessel occlusions in acute ischaemic sroke (TREVO 2): a randomised trial. Lancet 380:1231-1240

14. Pfefferkorn T, Holtmannspötter M, Schmidt C et al (2010) Drip, ship, and retrieve: cooperative recanalization therapy in acute basilar artery occlusion. Stroke 41:722-726

15. Rha J-H, Saver JL (2007) The impact of recanalization on ischemic stroke outcome: a meta-analysis. Stroke 38:967-973

16. Riedel $\mathrm{CH}$, Jensen U, Rohr A et al (2010) Assesment of thrombus in acute middle cerebral artery occlusion using thin-slice nonenhanced computed tomography reconstructions. Stroke 41:1659-1664

17. San Roman L, Obach V, Blasco J et al (2012) Single-center experience of cerebral artery thrombectomy using the TREVO device in 60 patients with acute ischemic stroke. Stroke 43:1657-1659

18. Smith WS, Sung G, Saver J et al (2008) Mechanical thrombectomy for acute ischemic stroke: final results $f$ the Multi MERCI trial. Stroke 39:1205-1212

19. Saver JL, Jahan R, Levy El et al (2012) Solitaire flow retoration device versus the merci retriever in patients with acute ischaemic stroke (SWIFT): a randomised, parallel-group, non-inferority trial. Lancet 380:1241-1249

20. Taschner CA, Treier M, Schumacher M et al (2011) Mechanical thrombectomy with the penumbra recanalization device in acute ischemic stroke. J Neuroradiol 38:47-52

21. Zeumer H, Hacke W, Kolmann HL, Poeck K (1982) Lokale Fibrinolysetherapie bei Basilaristhrombose. Dtsch Med Wochenschr 107:728-731 